\title{
Aperiodic Tilings with One Prototile and Low Complexity Atlas Matching Rules
}

\author{
D. Fletcher
}

Received: 26 March 2010 / Revised: 30 June 2010 / Accepted: 14 July 2010 /

Published online: 4 August 2010

(C) Springer Science+Business Media, LLC 2010

\begin{abstract}
We give a constructive method that can decrease the number of prototiles needed to tile a space. We achieve this by exchanging edge-to-edge matching rules for a small atlas of permitted patches. This method is illustrated with Wang tiles, and we apply our method to present via these rules a single prototile that can only tile $\mathbb{R}^{3}$ aperiodically, and a pair of square tiles that can only tile $\mathbb{R}^{2}$ aperiodically.
\end{abstract}

Keywords Tiling $\cdot$ Matching rules $\cdot$ Atlas $\cdot$ Prototile

\section{Introduction}

The field of aperiodic tilings was created by Berger's discovery [2] of a set of 20,426 square tiles which could only tile the plane in a non-repeating global structure. Unsurprisingly there has been some interest on how far this number could be decreased. The number of tiles was reduced over time, to 6 square tiles by Robinson [13] in 1971, and, relaxing to non-square tiles, to 2 tiles by Penrose [12] two years later. This led naturally to serious consideration about the possible existence of a single aperiodic tile (a monotile).

While a simple example has not been forthcoming, by relaxing requirements on the monotile (such as being defined by shape alone, or connectedness) development has occurred. In [15] Socolar studied a more general problem, ' $k$-isohedral' monotiles, which would have an aperiodic monotile as a limiting case. Relaxing conditions on edge-colouring or non-connected tiles provided partial positive results. Myers has produced many examples of monotiles with high isohedral numbers, such as 10-isohedral polyhexagons and 6-isohedral polyominoes [11]. In 1996 Gummelt [9] considered tiles that are allowed to overlap, and produced a decorated tile which

\footnotetext{
D. Fletcher $(\bowtie)$

University of Leicester, Leicester, UK

e-mail: df67@leicester.ac.uk
} 
could force aperiodicity. Most recently in early 2010, Socolar and Taylor [16] produced a disconnected tile that could force aperiodicity.

This paper extends work by Goodman-Strauss on 'atlas matching rules'. In an aside in [7], Goodman-Strauss describes how by requiring a tiling be covered by a suitable finite atlas of permitted bounded configurations, a domino can serve as a monotile. The aside only produced a weak upper bound on the size of the atlas, but Goodman-Strauss postulated the bound could be reduced considerably.

In Sect. 2 we define our uses of the terms prototile, tiling and (strong) aperiodicity, and formalise the concepts of matching rules based on colours (including an generalisation) or atlases. We then describe a method of altering coloured matching rules to atlas matching rules with very small patches. If two or more of the tiles have the same shape, the number of prototiles needed is decreased. This method can be applied to tilings in general, not just aperiodic ones. We will restrict ourselves to connected prototiles in this paper for clarity, but the general method can be applied to disconnected prototiles as well.

In Sect. 3 we use our method to construct a pair of square tiles which can only tile $\mathbb{R}^{2}$ aperiodically, and a single cubic tile that can only tile $\mathbb{R}^{3}$ in a (strongly) aperiodic manner. Further improvements to the method are described in Sect. 4.

\section{The Atlas Matching Rule Construction}

We shall describe the basic definitions we will be using in this paper. For more details on this field we suggest Sadun's book [14]. For clarity we will be limiting the spaces we are tiling to $\mathbb{R}^{n}$ for some $n \in \mathbb{N}$. With minor alterations the method will work in any homogeneous space (for example hyperbolic space $\mathbb{H}^{n}$ ).

Let $\mathcal{P}$ be a finite set of compact subsets of $\mathbb{R}^{n}$, each the closure of its interior. Denote these subsets as prototiles. In this paper we require that prototiles have a cell complex as their boundary, and are connected. Thus there are vertices (or 0 -facets). Similarly on the boundary of the prototile there are edges (or 1-facets), faces and so on, up to $(n-1)$-facets.

Let $G$ be a group of isometries of $\mathbb{R}^{n}$, which includes all translations of $\mathbb{R}^{n}$. The groups we will be using most in this paper are the group of translations $G_{\operatorname{Tr}}$ and the group of all isometries $G_{I}$. We will be using these groups to define allowable isometries from prototiles to tiles.

Define $g(U)$ for some $U \subseteq \mathbb{R}^{n}$ and $g \in G$ as the set of points $p \in \mathbb{R}^{n}$ such that $g^{-1}(p) \in U$. For a given set of prototiles $\mathcal{P}$ and a group of isometries $G$, define a tile $t$ as the image $g(P)$, for some $g \in G, P \in \mathcal{P}$. A tile inherits the vertices, edges and other facets from its prototile. A patch for $\mathcal{P}$ is a set of tiles with pairwise disjoint interiors and the support of a patch is the union of its tiles. A tiling $T$ with prototiles $\mathcal{P}$ is a patch with support $\mathbb{R}^{n}$. We shall refer to the support of a prototile $P$ as $\operatorname{supp}(P)$.

There are a number of distinct uses in the field of aperiodic tilings of the term aperiodic. We shall use the following definitions, originating from [10] and in the format of [7];

Definition 1 A tiling $T \subset \mathbb{R}^{n}$ is weakly periodic if there exists an infinite cyclic subgroup $H$ of isometries of $\mathbb{R}^{n}$ such that $H T=T$ (i.e. for all $h \in H, h T=T$ ). A tiling 
Fig. 1 A tile $t$, and its 1-corona

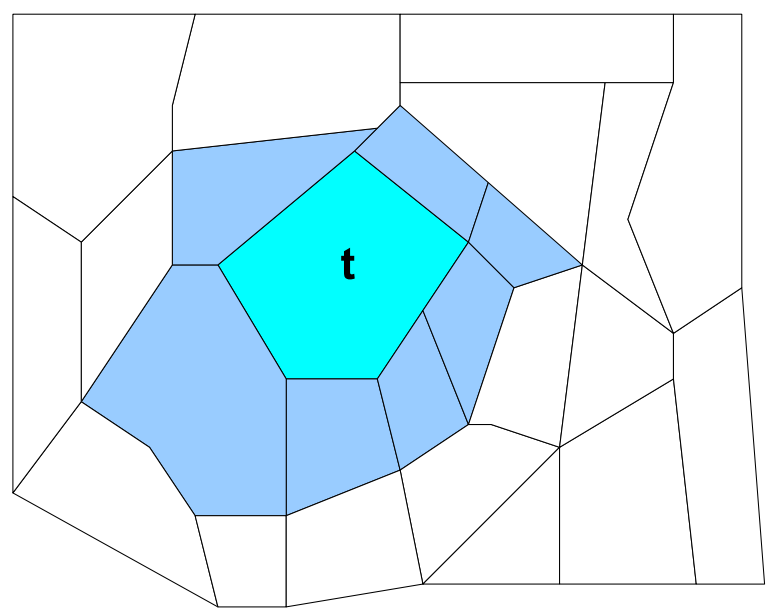

that is not weakly periodic is said to be strongly non-periodic. A set of prototiles $\mathcal{P}$ which can only construct strongly non-periodic tilings, is said to be strongly aperiodic.

A tiling $T$ of $\mathbb{R}^{n}$ is strongly periodic if there exists a discrete subgroup $H$ of isometries of $\mathbb{R}^{n}$ with $\mathbb{R}^{n} / H$ compact and $H T=T$. A tiling that is not strongly periodic is weakly non-periodic. A set of prototiles is weakly aperiodic if it can only construct weakly non-periodic tilings.

Note that in two dimensions the two definitions of aperiodicity are equivalent (see Theorem 3.7.1 in [8] for proof). Thus we refer to 'aperiodicity' in two dimensions, and 'strong aperiodicity' in three or higher.

In this paper, we require that tiles meet in whole facets and that tiles are connected. Similar ideas can be applied to tiles which are disconnected.

We will be using patches defined by the '1-corona' about a tile $t$.

Definition 2 The '1-corona' of a tile $t$ is the set of tiles with non-empty intersection with $t$ (see [7]).

As an example, see Fig. 1. This picture shows a tile $t$, and its 1-corona (all marked tiles, including $t$ itself).

We now want to introduce the notion of 'colouring' a prototile's boundary, and hence all tiles produced from it. In this paper we only care about the highest dimensional parts of a boundary. Thus for 2 dimensional tiles we only care about edges of a tile, not vertices. For 3 dimensional tiles we only care about faces, not edges or vertices, and so on. Thus for an $n$ dimensional tile, we will assign to each $(n-1)$ dimensional facet of the boundary of the tile an element of a given set, as follows.

Let $\mathcal{P}$ be prototiles in $\mathbb{R}^{n}$. Construct a function $\lambda:\{(n-1)$-facets of $P \in \mathcal{P}\} \rightarrow C$ where $C$ is a non-empty set. A facet $x$ of the prototile $P$ is $c$-coloured if $\lambda(x)=c$.

Extend $\lambda$ to facets of any given tile $t=g(P)$ by $\lambda_{t}(x)=\lambda g^{-1}(x)$ for each $(n-1)$ facet $x$ of $t$. 
Definition 3 A coloured tiling $(T, \lambda)$ of $\mathbb{R}^{n}$ satisfies the identical facet (matching) rule if for all tiles $t_{1}, t_{2} \lambda_{t_{1}}(x)=\lambda_{t_{2}}(x)$ for each $(n-1)$-facet $x$ that $t_{1}$ and $t_{2}$ share.

This covers cases where two tiles 'match' if they have the same colour on the interior of their shared boundary (for example Wang tiles, which match when they share edges of a common colour).

We will be using a slightly more general version of this rule in the rest of this paper, which allows tiles to match under wider conditions, as follows.

Definition 4 A facet (matching) rule is a function on pairs of colours $r: C \times C \mapsto$ $\{0,1\}$ such that $r(x, y)=r(y, x)$ and $r(0,0)=1$. A coloured tiling $(T, \lambda)$ satisfies the facet (matching) rule $r$ if for all tiles $t_{1}, t_{2}$ (where $t_{1} \neq t_{2}$ ),

$$
r\left(\lambda_{t_{1}}(x), \lambda_{t_{2}}(x)\right)=1
$$

for all facets $x$ of $t_{1} \cap t_{2}$.

The obvious question is when do these facet matching rules coincide with matching rules defined only in terms of shape of tile boundary. As stated at the start of the section, we are only using connected tiles which match facet-to-facet.

Let us consider the matrix corresponding to the facet matching rule $r$, where $a_{i j}=$ $r\left(c_{i}, c_{j}\right)$ for some fixed enumeration of the colours which can be associated to the tiles' edges. Note that the matrix must be symmetric, since $r(x, y)=r(y, x)$.

We will assume colours have been made 'distinct' in the sense that for all $x, c_{i}$, $c_{j} \in C, r\left(c_{i}, x\right)=r\left(c_{j}, x\right)$ implies $i=j$. This condition is equivalent to having two different colours which match in precisely the same way (and can thus be identified together). In this matrix this corresponds to ensuring no row is a copy of another row, by removing rows until this is not the case. Similarly we will ignore colours which cannot match any colour, including themselves, because any tile with such a colour on its boundary cannot occur in a tiling satisfying that facet matching rule.

Then a matching rule $r$ can be expressed in terms of shape of tile boundary if and only if there is only one 1 entry in every row and column of its matrix.

We shall illustrate why this is true, starting with the two dimensional case. If you have two tiles with a common edge meeting at vertices $u, v$ then for a given curved edge on one tile, there exists precisely one curved edge which meets it at every point. Similarly in $\mathbb{R}^{n}$, for any $(n-1)$-facet on the boundary of a tile, there exists precisely one $(n-1)$-facet which can meet it. Thus for any coloured boundary facet, there is only one other colour of facet that can meet it. Thus any row or column of the matrix must have only one non-zero entry.

Let us consider some examples.

Example 1 Consider the facet rule matrix in the following figure.

This matrix has only one entry with the value 1 in each row, thus it can be expressed in terms of curved edges. One way of doing this would be to set $c_{1}$ as a straight edge, $c_{2}$ as a edge with a protrusion out from it, and $c_{3}$ as the unique edge that can fit to it. 


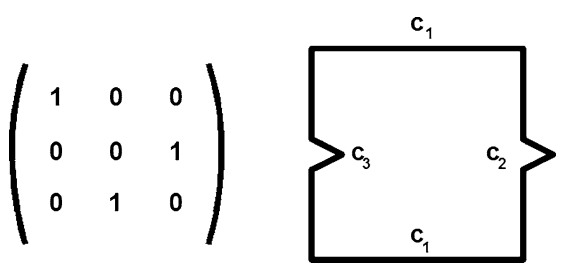

Example 2 Let the set of colours on a tiling be the set of cards in a normal 52 playing card deck.

Let $r\left(c_{i}, c_{j}\right)=1$ iff $c_{i}$ and $c_{j}$ are from the same suit, or have the same face value. Every colour matches to a unique subset of colours, thus we do not need to remove rows or columns from the matrix. Furthermore, each colour matches to 16 other colours (the 13 in the same suit, and 3 other cards with the same face value).

Thus this matching rule cannot be expressed in terms of curved edges.

We describe below a way of translating from a facet matching rule to a matching rule of the following type.

Definition 5 A tiling $T$ satisfies an atlas (matching) rule $\mathcal{U}$ if there exists a finite atlas of compact patches $U \in \mathcal{U}$ such that for every tile $t \in T$, there exists a patch $U_{t}$ about $t$ (with $t$ being in the strict interior of $U_{t}$ ) such that $U_{t}=g(U)$ for some $g \in G_{\operatorname{Tr}}$ and $U \in \mathcal{U}$.

A 1 -corona atlas rule is an atlas rule where every patch $U \in \mathcal{U}$ is the 1-corona of some tile $t$.

Definition 6 A tiling $T$ is a $(\mathcal{P}, G, \lambda, r)$-tiling if it has a prototile set $\mathcal{P}$ with allowable isometries $G$ and colouring $\lambda$, and satisfies the facet rule $r$.

A tiling $T$ is a $(\mathcal{X}, G, \mathcal{U})$-tiling if it has a prototile set $\mathcal{X}$ with allowable isometries $G$, and satisfies the atlas matching rule $\mathcal{U}$.

A tiling $A$ is locally derivable from a tiling $B$ if there exists a length $R$ such that, if $z_{1}, z_{2} \in \mathbb{R}^{d}$ and $A-z_{1}$ agrees with $A-z_{2}$ on a ball of radius $R$ around the origin, then $B-z_{1}$ agrees with $B-z_{2}$ on a ball of radius 1 around the origin. Thus the tile at a point $z$ in $B$ depends only on a finite patch around $z$ in $A$. If $B$ is locally derivable from $A$ and $A$ is locally derivable from $B$, then $A$ and $B$ are said to be mutually locally derivable (MLD) tilings. (The definition of MLD originates in [1], but we are using the equivalent variation found in [3].)

Theorem $1 A\left(\mathcal{P}, G_{\operatorname{Tr}}, \lambda, r\right)$-tiling $T$ is $M L D$ to a $\left(\mathcal{X}, G_{I}, \mathcal{U}\right)$-tiling for some 1 corona atlas rule $\mathcal{U}$ and a prototile set $\mathcal{X}$ with $|\mathcal{X}| \leq|\mathcal{P}|$.

Construction 1 Take $\mathcal{P}$ and partition it into a set of equivalence classes $\mathcal{P}=\coprod \mathcal{P}_{s}$, $s \in\{1, \ldots, m\}$ where $P_{i} \sim P_{j}$ iff $\operatorname{supp}\left(P_{i}\right)=\operatorname{supp}\left(P_{j}\right)$ up to the action of an element of $G_{\mathrm{Tr}}$. For each $\mathcal{P}_{S}$, let $\Psi_{s}$ be the group of automorphisms of any of the prototiles $P \in \mathcal{P}_{\text {s }}$.

Enumerate the elements of $\mathcal{P}_{s}$ as $P_{1}^{s}, \ldots, P_{r}^{s} \in \mathcal{P}_{s}$.

Choose the smallest $k$ you can so as to construct an injective function $e_{s}: \mathcal{P}_{s} \rightarrow$ $\left\{\left(P_{i}^{s}, \psi_{j}\right) \mid 1 \leq i \leq k, \psi_{j} \in \Psi_{s}\right\}$. Define $\mathcal{X}_{s}=\left\{P_{1}^{s}, \ldots, P_{k}^{s}\right\}$. 
We now have a construction taking prototiles $P_{i}^{s} \in \mathcal{P}_{s}$ to ordered pairs of a prototile from $\mathcal{X}_{s}$ and an automorphism of that prototile. Observe that $\mathcal{X}_{s}$ is a subset of $\mathcal{P}_{s}$.

Proof of Theorem 1 Define a new prototile set $\mathcal{X}=\mathcal{X}_{1} \cup \cdots \cup \mathcal{X}_{m}$, where $\mathcal{X}_{s}$ is as just defined. Let the set of allowable functions from the prototiles into $\mathbb{R}^{n}$ be $G_{I}$, instead of $G_{\mathrm{Tr}}$. Take the set of allowable 1-coronas in the $\left(\mathcal{P}, G_{\mathrm{Tr}}, \lambda, r\right)$-tiling $T$, and replace every tile originating from a translation of a prototile $P_{a}^{s} \in \mathcal{P}_{s}$ with $\psi_{j}\left(P_{i}^{s}\right)$, with $\psi_{j}$ and $P_{i}^{s}$ originating from $e_{s}\left(P_{a}^{s}\right)=\left(P_{i}^{s}, \psi_{j}\right)$. This will give you a set of 1 -corona patches of $\mathcal{X}$. Use this set as the atlas rule $\mathcal{U}$ for $\mathcal{X}$.

$T$ has facet rules, which are intrinsic to the set of allowable first coronas (since the set of allowable first coronas list what boundaries are allowed to meet each other). Since our definition of $\mathcal{X}$ and its atlas correspond to the first coronas of tiles in $T$, with $P_{a}^{s}$ replaced by $g_{j}\left(P_{i}^{s}\right)$, any tiling by $\mathcal{X}$ is MLD to a tiling from $\mathcal{P}$. Since $\left|\mathcal{X}_{s}\right| \leq\left|\mathcal{P}_{s}\right|$ we have $|\mathcal{X}| \leq|\mathcal{P}|$.

For reasons of clarity, we will give a concrete example of how to move from a given prototile $\widehat{P}$ in $\mathcal{P}$ to its image $g_{j}\left(\widehat{P}_{i}^{s}\right)$. Consider Fig. 2 . At the top of the picture we have a set of 13 prototiles, $\mathcal{P}$. The first step is to group the prototiles into sets (the $\mathcal{P}_{s}$ of the construction). A set must contain prototiles which have the same support up to translation, but cannot have more prototiles than there are automorphisms of that support. For example, with the rectangular tiles, there are four automorphisms which will send a rectangle onto itself. Thus the sets containing rectangles cannot contain more than four rectangles. (Note that the two $F$ shapes must be placed in different $\mathcal{P}_{s}$ sets, since they do not have the same support up to translation. In the final section we will describe a construction which can place them in the same $\mathcal{P}_{s}$ set.)

The second step is to take one of the new collections of tiles with the same support. In the figure we have taken one of the sets of rectangles. Then replace one of the tiles with a new prototile $P_{i}$. For each other prototile, take $P_{i}$ and apply a different automorphisms of the support of $P_{i}$ to associate an automorphism of $P_{i}$ to that prototile. Then substitute the new prototiles you have produced into the allowable 1-coronas of $\mathcal{P}$ to produce a tiling with less prototiles needed.

Corollary Take a prototile set $\mathcal{P}$ and partition it into a set of equivalence classes $\mathcal{P}=\coprod \mathcal{P}_{s}, s \in\{1, \ldots, m\}$ as in the previous construction. If there exists $\mathcal{P}_{s}$ such that $\left|\mathcal{X}_{s}\right|<\left|\mathcal{P}_{s}\right|$, there exists a prototile set (with atlas rules) which tiles $\mathbb{R}^{n}$ with less prototiles than $\mathcal{P}$.

Proof We know that $\left|\mathcal{X}_{s}\right|<\left|\mathcal{P}_{s}\right|$, thus $|\mathcal{X}|<|\mathcal{P}|$.

Remark This method of construction produces a prototile set with cardinality $\sum_{s=1}^{m}\left\lceil\frac{\left|\mathcal{P}_{s}\right|}{\left|\Psi_{s}\right|}\right\rceil$.

Remark $\mathcal{P}$ is (strongly) aperiodic iff $\mathcal{X}$ is (strongly) aperiodic. This is because every tiling in $\mathcal{X}$ is MLD to a tiling in $\mathcal{P}$, and strong aperiodicity is preserved under MLD equivalency. 


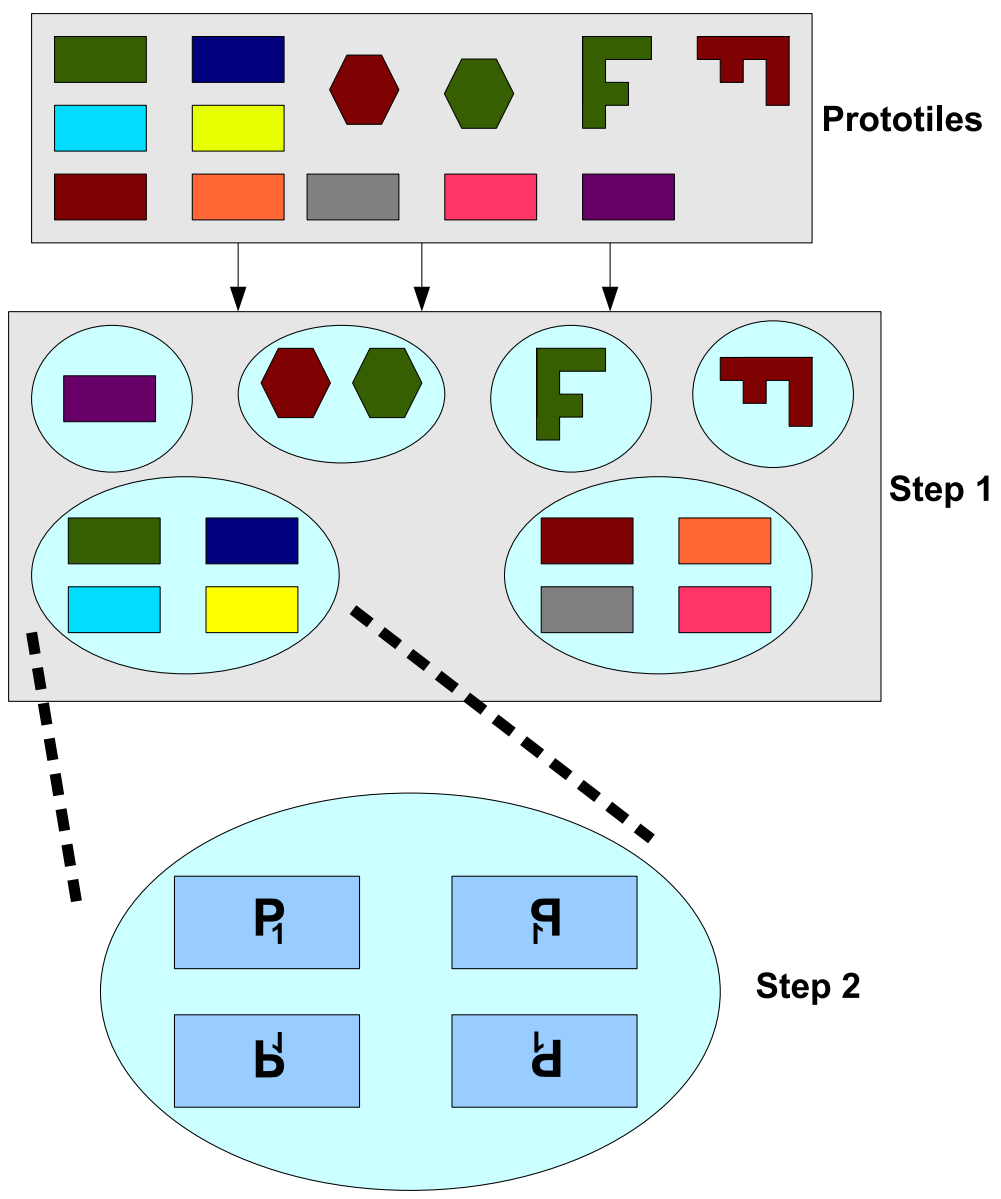

Fig. 2 Applying the construction to a set of prototiles

\section{Motivating Examples and Aperiodicity}

Example 3 For a simple illustration of the method, let us consider a tiling of the plane by 13 Wang prototiles (unit squares with matching rules defined by matching coloured edges) as given in $[4,5,17]$. Label the Wang prototiles as $\left\{Q_{1}, \ldots, Q_{13}\right\}$. We can apply the above construction to get a function from $\left\{Q_{j}\right\}_{j=1}^{13}$ to $\left\{\left(P_{i}, r\right) \mid 1 \leq\right.$ $\left.i \leq 2, r \in D_{4}\right\}$, where $D_{4}$ is the group of symmetries of the square.

For example, enumerate the symmetries of the square as $\left\{r_{1}, r_{2}, \ldots, r_{8}\right\}$. Then such a function could send $\left\{Q_{j} \mid 1 \leq j \leq 8\right\}$ to $\left(P_{1}, r_{j}\right)$, and the remaining tiles $\left\{Q_{j} \mid 9 \leq\right.$ $j \leq 13\}$ to $\left(P_{2}, r_{j-8}\right)$. The result is shown in Fig. 3 , for a small patch of the tiling.

As is common with Wang tiles, the colouring of $\left\{Q_{j}\right\}_{j=1}^{13}$ is represented as actual colours superimposed onto the tile. For the diagram we represent the change of prototile set from $\left\{Q_{j}\right\}_{j=1}^{13}$ to $\left\{P_{1}, P_{2}\right\}$ by adding a label to $P_{1}$ and $P_{2}$, which looks like their alphabetical symbols. This label will be visually affected by the automorphisms 
Fig. 3 Top picture shows a tiling with prototiles $Q_{1}, \ldots, Q_{13}$ with facet matching rules, and translation as an isometry group. The bottom picture uses a two element prototile set, with rotations, reflections and translations as a isometry group
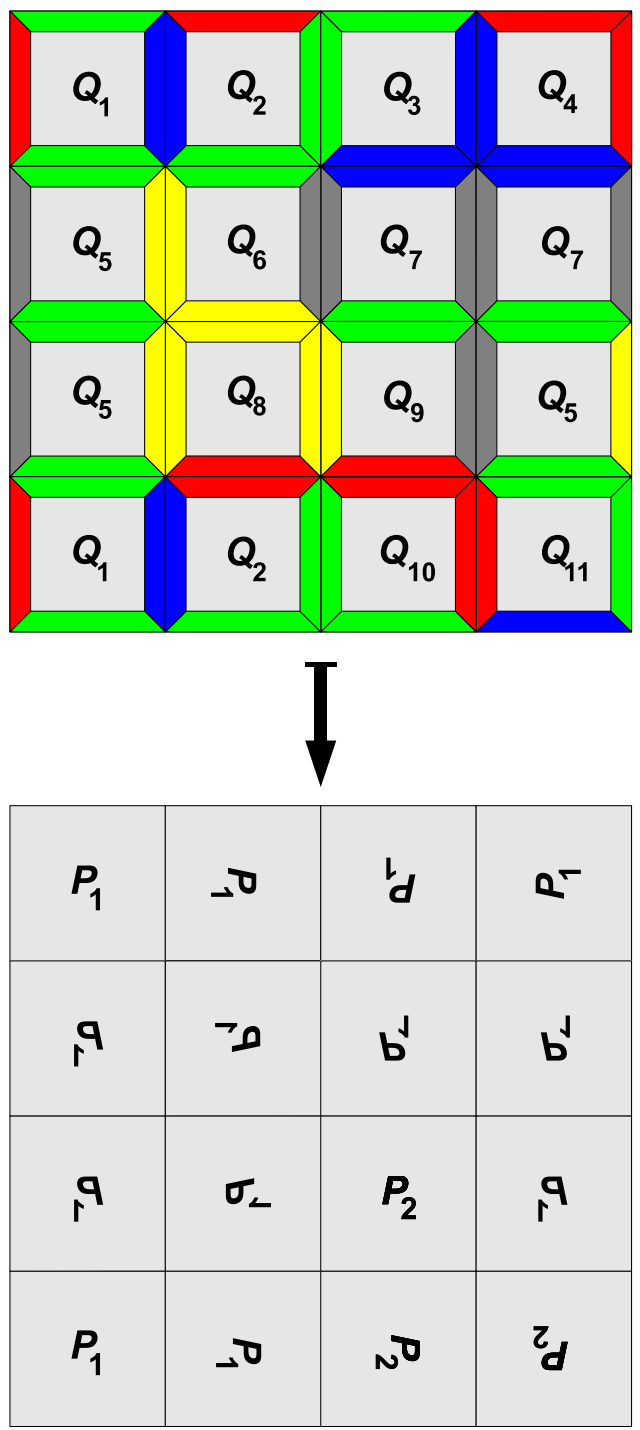

of $P_{1}$ and $P_{2}$. This is solely for the diagram, and is intended to assist the reader in distinguishing between the various automorphisms of $P_{1}$ and $P_{2}$.

Next we will consider a 3 dimensional example. As mentioned before, aperiodicity in higher dimensions is more complicated and we have to worry about weak and strong aperiodicity. We will solve this problem by extending a known example of a prototile set which only tiles in a strongly aperiodic manner.

Example 4 Consider Kari's Wang cube prototiles, $W$ [6]. This is a set of 21 unit cube prototiles with facet matching rules, where every tiling of $\mathbb{R}^{3}$ by $W$ is strongly aperiodic. 
Choose a unit cube prototile $A$.

Since the set of isometries of the cube (and thus $A$ ) is of cardinality 48, we can choose 21 unique isometries of $A, i_{k}, 1 \leq i \leq 21$. We use the method in Construction 1 to replace $P_{k} \in W$ with $i_{k}(A)$.

Thus we have an aperiodic protoset with one prototile which is MLD to Kari's Wang Cubes. Note that we have lost the property of matching rules being determined on faces, and replaced them with a set of legal one corona patches (which cannot be rotated or reflected, of course). We have also had to broaden the set of allowable mappings of the prototiles into the tiled space, from translations to translations and rotation/reflections.

\section{Further Improvements}

Remark The construction can be further improved, by partitioning $\mathcal{P}$ into equivalence classes based on what prototiles have the same support up to isometry, not just translation.

Let $T$ be a ( $\left.\mathcal{P}, G_{\mathrm{Tr}}, \lambda, r\right)$-tiling as in Construction 1 . If there is a prototile $P_{i} \in \mathcal{P}$ whose support is a non-trivial isometry of another prototile $P_{j}$ (where $i \neq j$ ), then the resulting $\left(\mathcal{X}, G_{I}, \mathcal{U}\right)$-tiling may have less prototiles than one originating from Construction 1. An example of this is in Fig. 2, where the two $F$-shape tiles have equivalent support up to rotation.

Construction 2 Partition $\mathcal{P}=\coprod^{s}, s=\{1, \ldots, p\}$ where $P_{i} \sim P_{j}$ iff $\operatorname{supp}\left(P_{i}\right)=$ $\operatorname{supp}\left(P_{j}\right)$ up to the action of an element of $G_{I}$.

Further partition $\mathcal{P}^{s}=\coprod \mathcal{P}_{t}^{s}, t=\{1, \ldots, q\}$ where $P_{a}^{s} \sim P_{b}^{s}$ iff $\operatorname{supp}\left(P_{a}^{s}\right)=$ $\operatorname{supp}\left(P_{b}^{s}\right)$ up to the action of an element of $G_{\mathrm{Tr}}$.

This two-stage partitioning gives us a collection of equivalence classes $\left(\coprod_{s, t} \mathcal{P}_{t}^{s}\right)$ as per the first construction. Additionally we know that there exist isometries in $G_{I}$ from elements of $\mathcal{P}_{i}^{s}$ to elements of $\mathcal{P}_{j}^{s}$. Take the $\mathcal{P}_{t}^{s}$ with the largest cardinality and denote it $\mathcal{P}_{T}^{s}$. From the definition of $\mathcal{P}^{s}$ there exists an isometry $\alpha_{P_{i} P_{j}}$ such that $\alpha_{P_{i} P_{j}}\left(\operatorname{supp}\left(P_{i}\right)\right)=\operatorname{supp}\left(P_{j}\right)$. Furthermore we know that an given isometry can only take elements from one set $\mathcal{P}_{i}^{S}$ to $\mathcal{P}_{T}^{S}$ (by definition of equivalence class). Thus we can replace any prototile in $\mathcal{P}_{t}^{s}$ with a unique isometry of a prototile in $\mathcal{P}_{T}^{s}$, since $\left|\mathcal{P}_{t}^{s}\right| \leq\left|\mathcal{P}_{T}^{s}\right|$

By applying the previous construction to $\mathcal{P}_{T}^{s}$, we can get a minimal uncoloured prototile set $\mathcal{X}^{s}$ that can be used to translate prototiles in $\mathcal{P}_{T}^{s}$, and hence $\mathcal{P}^{s}$, to atlas rules.

Example 5 Take a prototile set $\mathcal{T}$ of equilateral triangles, as shown in Fig. 4. The prototiles have two different orientations, and three (could be up to six) colours.

We partition $\mathcal{T}$ into $\mathcal{T}=\mathcal{T}_{1}$, since all prototiles in $\mathcal{T}$ have the same support, up to isometry. We then further partition $\mathcal{T}_{1}=\mathcal{T}_{1}^{1} \amalg \mathcal{T}_{1}^{2}$, where $\mathcal{T}_{1}^{1}$ is the set of prototiles with point upwards, and $\mathcal{T}_{1}^{2}$ is the set of prototiles with point downwards. Denote the first prototile of $\mathcal{T}_{1}^{1}$ as $t_{1}$. Applying the first construction to $\mathcal{T}_{1}^{1}$ gives you $f\left(P_{i} \in\right.$ $\left.P_{1}^{1}\right)=d_{i}\left(t_{1}\right)$, for $d_{i} \in D_{3}$, and $f\left(P_{i} \in P_{1}^{2}\right)=\operatorname{rot} \frac{\pi}{3}\left(d_{i}\left(t_{1}\right)\right)$. While this is sufficient 


\section{$\boldsymbol{T}$, the old prototile set}
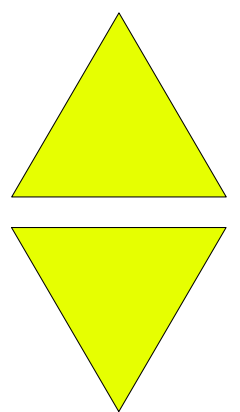
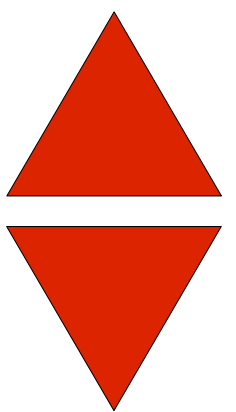
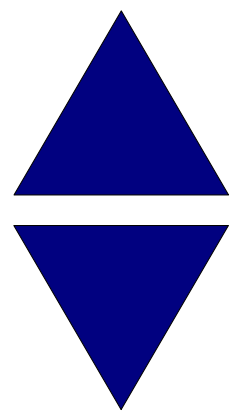

$\boldsymbol{X}$, the new prototile

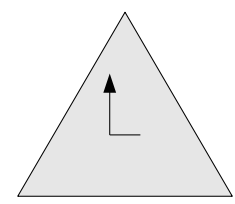

Fig. 4 New and old prototile set

to define the tiling, it has the problem that any picture of the tiling needs to include information about the isometries used for each tile. Thus we replace $t_{1}$ with a tile $x$ with an uncoloured boundary, but with a coloured interior which is not preserved under any non-identity element of $D_{3}$.

Acknowledgements Thanks are due to Chaim Goodman-Strauss, Joshua E.S. Socolar and Edmund Harriss for helpful conversations on this paper. My supervisor John Hunton has assisted considerably with improving the readability of this paper. We also thank the University of Leicester and EPSRC for a doctoral fellowship. The results of this article will form part of the author's PhD thesis.

\section{References}

1. Baake, M., Schlottmann, M., Jarvis, P.D.: Quasiperiodic tilings with tenfold symmetry and equivalence with respect to local derivability. J. Phys. A 19, 4637-4654 (1991). MR1132337

2. Berger, R.: The undecidability of the domino problem. Mem. Am. Math. Soc. 66 (1966)

3. Clark, A., Sadun, L.: When shape matters: deformations of tiling spaces. Ergod. Theory Dyn. Syst. 26, 69-86 (2006). doi:10.1017/S0143385705000623

4. Culik, K. II: An aperiodic set of 13 Wang tiles. Discrete Math. 160(1-3), 245-251 (1996)

5. Kari, J.: A small aperiodic set of Wang tiles. Discrete Math. 160(1-3), 259-264 (1996)

6. Kari, J., Culik, K. II: An aperiodic set of Wang cubes. J. Univers. Comput. Sci. 1(10), 675-686 (1995)

7. Goodman-Strauss, C.: Open questions in tilings. January 10, 2000. http://comp.uark.edu/ strauss/ papers/survey.pdf

8. Grünbaum, B., Shepherd, G.C.: Tilings and Patterns. W.H. Freeman, San Francisco (1987)

9. Gummelt, P.: Penrose tilings as coverings of congruent decagons. Geom. Dedic. 62(1) (1996). doi:10.1007/BF00239998

10. Mozes, S.: Aperiodic tilings. Invent. Math. 128, 603-611 (1997)

11. Myers, J.: http://www.srcf.ucam.org/ jsm28/tiling/

12. Penrose, R.: Role of aesthetics in pure and applied research. Bull. Inst. Math. Appl. 10, 266-271 (1974)

13. Robinson, R.M.: Undecidability and nonperiodicity for tilings of the plane. Invent. Math. 12(3), 177209 (1971)

14. Sadun, L.: Topology of Tiling Spaces. University Lecture Series, vol. 46 (2008)

15. Socolar, J.E.S.: More ways to tile with only one shape polygon. Math. Intell. 29(2) (2007). doi:10.1007/BF02986203

16. Socolar, J.E.S., Taylor, J.M.: An aperiodic hexagonal tile. http://arxiv.org/abs/1003.427

17. Wang, H.: Proving theorems by pattern recognition II. Bell Syst. Tech. J. 40(1), 1-41 (1961) 